الأدلة الربوبية والأحيائية والمعدنية على حدث فقدان الأوكسجين المحيطي -2 لتكوين

$$
\text { كولنيري (التوروني المبكر) في شمال شرقي العراق }
$$

فلاح عبد المعماري

$$
\begin{aligned}
& \text { صفوان فتحي اللهيبي علي إسماعيل الجبوري } \\
& \text { قسم علوم الأرض إعل النوري } \\
& \text { كلية العلوم } \\
& \text { جامعة الموصل كلئل }
\end{aligned}
$$$$
\text { تاريخ الاستلام 2020/9/20 تاريخ القبول 2020/11/21 }
$$

\title{
الملخص
}

درس تكوين كولنيري (التوروني المبكر) في ثلاثة مقاطع سطحية (ازمر وديجلة ودوكان) شمال شرقي العراق.

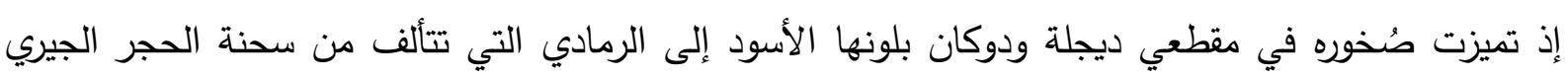

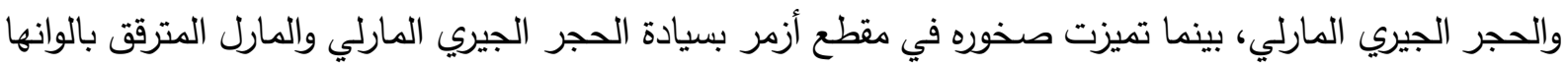

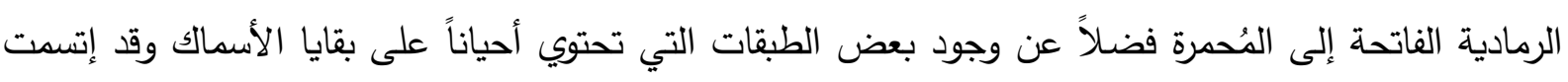

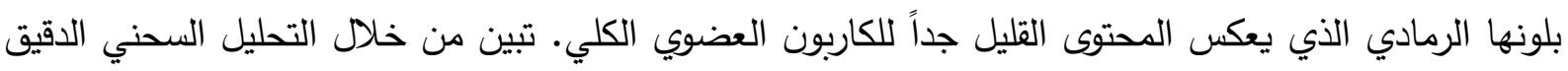

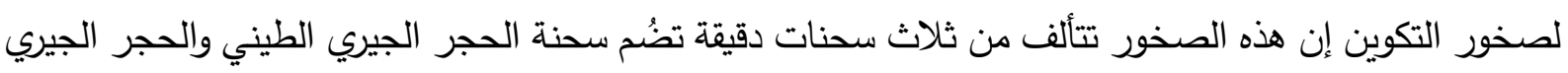

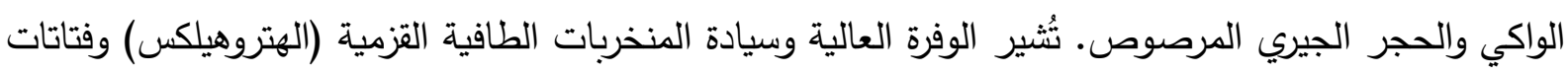
الأصداف النحيفة (الفلمنت) لاسيما في سحنة الحجر الجيري المرصوص إلى حدث الحئ إنحراف الهتيروهلكيس

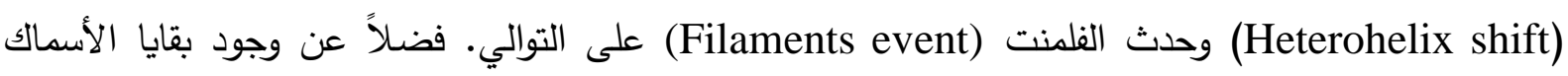

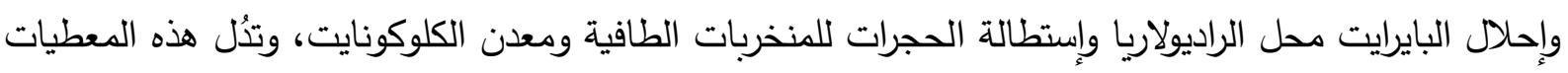

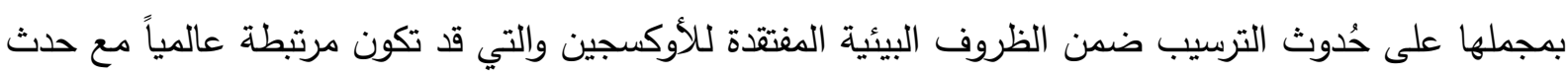
فقدان الأوكسجين الدحيطي-2 العالمي عند الحد الفاصل بين السينوماني -التوروني. الكلمات الدالة: تكوين كولنيري، حدث فقدان الأوكسجين المحيطي-2، التوروني المبكر.

\section{Sedimentological, Paleontological and Mineralogical Evidences for Oceanic Anoxic Event-2 (OAE-2) in the Gulneri Formation (Early Turonian), Northeastern Iraq}

\section{Safwan Fathi Al-Lhaebi Ali Ismail Al-Jubory \\ Geology Department \\ College of Science \\ University of Mosul}

Falah Abed Al-Miamary

\section{ABSTRACT}

In the current work, the Gulneri Formation (Early Turonian) is studied in three surface sections (Degala, Dokan and Azmir) from northeastern Iraq. The formation is characterized by grey to black color limestone and marly limestone in the Degala and 
Dokan sections, whereas in Azmir section, fissile marl and marly limestone with pale to reddish color are dominated in addition to few beds, which occasionally contain fish remains, with distinct gray color reflecting the very few amount of total organic carbon in these beds. Microfacies analysis revealed that the formation consists of three microfacies: mudstone, wackestone and packstone. The predominance of dwarfish planktonic foraminifera (Heterohilex) and thin shell filaments particularly in packstone microfacies represent Heterohelix shift event and filament event respectively. In addition to these events, fish remains, radiolarian pyritization, planktonic foraminiferal chambers elongation and glauconite are all refer to anoxic environmental conditions that may have been coincided with the Global Cenomanian-Turonian Oceanic Anoxic Event (OAE-2).

Keywords: Gulneri Formation, oceanic anoxic event-2 (OAE-2), Early Turonian.

\section{المقدمة}

إستخدم حدث فقدان الأوكسجين المحيطي (Oceanic Anoxic Event) لوصف الظواهر التي تتميز بترسبات تبدو ذات إنتثار عالمي ضمن التتابعات اللجية لعمر الأبتيان-الألبيان والسينوماني-التوروني التي تحتوي

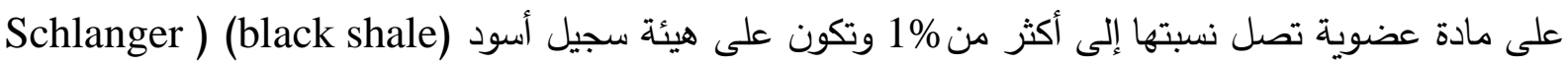
and Jenkyns, 1976 ). ويطلق على الحدث الذي يضُم الفترة الزمنية الواقعة بين السينوماني-التوروني بحدث فقدان الأوكسجين المحيطي-2 (Cenomanian-Turonian) مثالاً نموذجياً لأحداث فقدان الأوكسين في دهر الحياة المتوسطة وذلك بسبب الإنتشار الواسع للترسبات الغنية بالكاربون العضوي على مقياس عالمي (Kolonic et al., 2005; Smrzka et al., 2017). وقد أدى هذا لهال الحدث الى حصول إضطرابات كبيرة وسريعة بغاز ثنائي أوكسيد الكاربون الجوي ومحيطات العصر الطباشيري

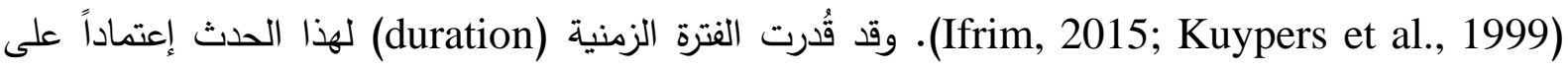
الطباقية الحياتية بفترة نصف مليون سنة (Caron et al., 1999) في توفي إيستبورن، إنكلترا

Arthur et al., ) وكذلك في تسعة مقاطع عالمية لحوض محيط الأطلسي ومحيط التثيس (Paul et al., 1999)

يعد هذا الحدث (OAE-2) واحدا من أهم أحداث فقدان الأوكسجين المحيطي الذي تم توثيقه في تاريخ

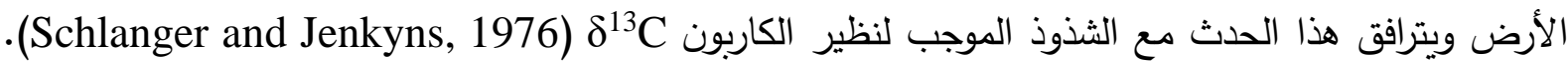

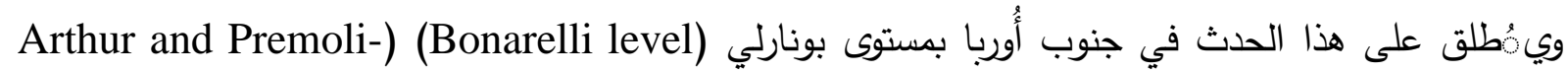
Crumière, 1990 (Silva, 1982 (Thomel Level) ويطلق عليه في جنوب شرق فرنسا بمستوى ثوميل هيل .(in Takashima et al., 2009

يتميز هذا الحدث بصورة عامة بالتقدم البحري العالمي والإرتفاع السريع لمنسوب سطح البحر، إذ وصل

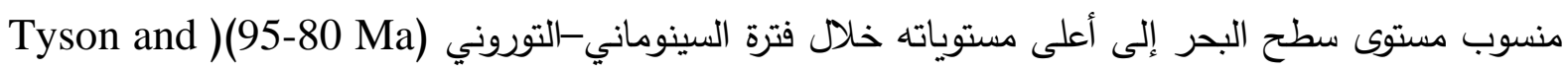
(Pearson, 1991; Keller and Pardo, 2004 . وكذلك تميز هذا الحدث بقصر الفترة الزمنية وبقلة نسبة Soua , 2010; Soua et al., (laminated deposits) وندرة الأحياء القاعية (الترسيب والترسبات المتصفحة (1a) 
يُعد تكوين المناطق النارية الكبيرة (Large igneous provinces) لاسيما (Caribbean Plateau) و (Ontong Java) و (Madagascar Plateaus) التي بدأت بالتطور خلال فترة السينوماني-التوروني مُؤشراً قوياً Snow et al., 2005; Kuroda et al., 2007; Turgeon and ) 2لبداية إنطلاق حدث فقدان الأوكسجين .(Creaser, 2008

وتنفرد فترة السينوماني-التوروني عن باقي الفترات الزمنية بكونها فترة دفيئة جداً (Pucéat et al., 2003) وذلك لإرتفاع نسبة غاز ثنائي أوكسيد الكاربون CO في الغلاف الجوي بنسبة تصل الى أكثر من أربعة مرات عن الوقت الحالي (Berner, 2001). فضلاً عن حدوث الإنغمار للعديد من المنصات الكاربوناتية خلال دهر الحياة المتوسطة. وقد شُخصت الترسبات الغنية بالكاربون العضوي تحت ظروف (dysoxic) و (anoxic) عند حدود Schlanger and ) السينوماني-التوروني في العديد من المواقع القارية حول العالم وكذلك في البحار العميقة Jenkyns, 1976; Jenkyns, 1980; Hasegawa, 1997; Sageman et al., 1998; Sugarman et al.,

توجد أراء عديدة وجدال واسع حول طبيعة الظروف التي أدت الى ترسيب السجيل الأسود سيما في فترة

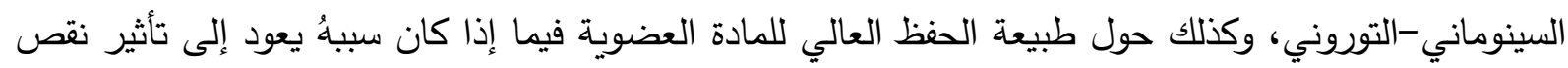
الاوكسجين للمياه العميقة او يعود سببه الى الانتاجية العالية (Stein, 1990). ولقد إفترض الباحث نفسُه أُنموذجين متطرفين لبيئتين لتوضيح وجود السجيل الأسود خلال فترات أحداث فقدان الأوكسجين (OAEs)، وهما موديل الحصر أو الحفظ (Stagnation or Preservation model) وموديل نطاق نقص الأوكسجين أو الإنتاجية

(الثكل 1) (Productivity model)

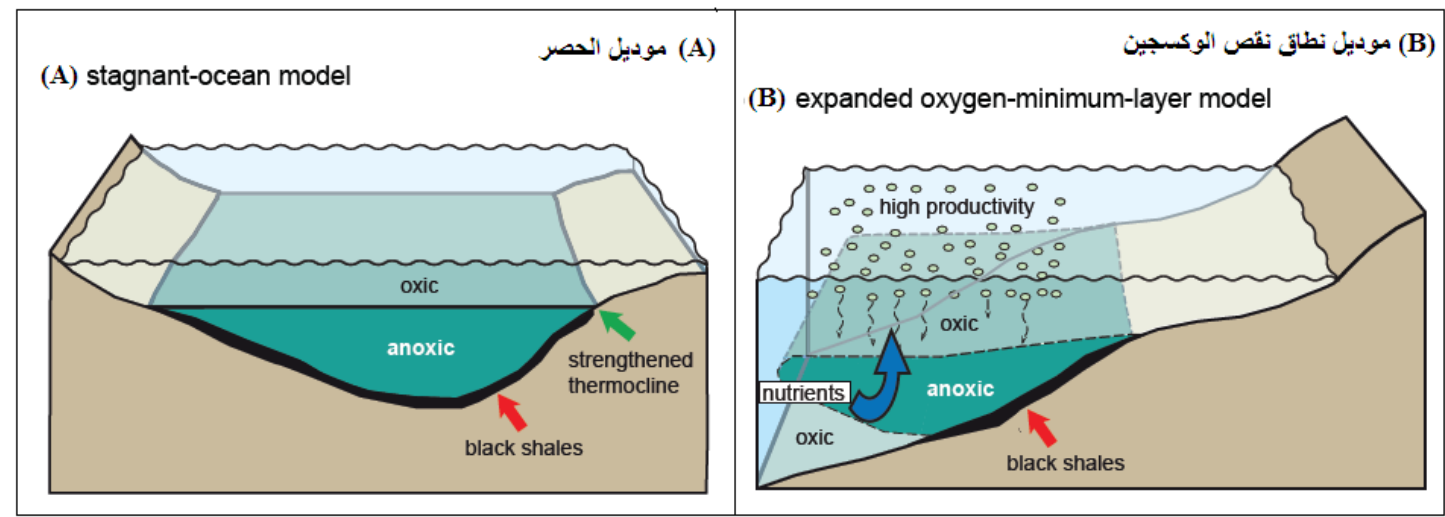

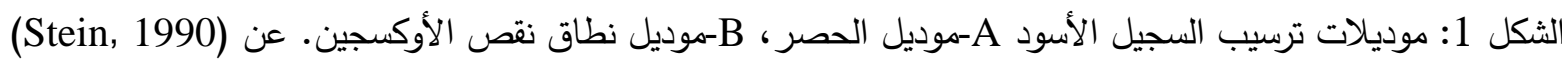
محور من قبل (Takashima et al., 2006).

ويشمل البحث الحالي إجراء دراسة تفصيلية للطبيعة الصخرية والسحنية من خلال الوصف الحقلي والدراسة البتروغرافية ودراسة السحنات الصخرية والدقيقة لصخور التكوين. وتهدف الدراسة الى إستتباط الأدلة الرسوبية والأحيائية والمعدنية التي تدل على ظروف فقدان الأوكسجين المحيطي أثناء فترة ترسيب التكوين.

\section{الوضع الجيولوجي وموقع منطقة الدراسة}

وصف (Bellen et al., 1959) تكوين كولنيري السجيلي لأول في نطاق الطيات العالية قرب موقع سد دوكان في منطقة السليمانية شمال شرقي العراق. وتتألف صخور التكوين من السجيل الجيري ذي الطبقات الرقيقة 
السوداء اللون التي تحتوي على معدني الكلوكونايت والكولوفين في جزئه السفلي وحدد عمر التكوين بالتوروني المبكر • يتراوح سمك التكوين بين 1.1-1.2 متر ، ويمثل سطحا التماس السفلي مع تكوين دوكان والعلوي مع تكوين كوميتان سطحي عدم توافق (Buday, 1980). يدل المحتوى العالي للمواد القيرية والمتحجرات القزمية ( Dwarf (fossils ويعد (Karim et al., 2013) اول من سجل مكافئ تكوين كولنيري ( Equivalent of Gulneri) (Formation عند الحد الفاصل بين السينوماني-التوروني في شمال شرقي السليمانية عند طيتي أزمر (Azmir) وكوجة أو كوزا (Goizha) بين تكوينى بالامبو وكوميتان. وقد أشاروا إلى أن هذه الصخور تمتلك صفات صخارية تختلف عن الصفات الصخارية لتكوين كولنيري من حيث خلوها من السجيل الأسود الذي يعود إلى تكوين كولنيري حسب (Bellen et al., 1959). وقد تم تسمية المقطع المذكور بمقطع أزمر في الدراسة الحالية. وكما درس (Karim and Khanaqa, 2017) الطباقية والتركيبية للجزء الثمالي الثرقي لطية بيرا مكرون في منطقة السليمانية شمال شرقي العراق، وحددا عمر تكوين كولنيري إعتماداً على متحجرات النانو بالسينوماني المتاخر -التوروني المبكر ، وقد أشاروا الى إمكانية إستخدام تكوين كولنيري الذي يمتد سمكه بين 2-4 متر كطبقة دالة لتمييز تكوين بالامبو بضمنه تكوين دوكان عن تكوين كوميتان حقليا بسبب صخاريته المتميزة. وقد أشار (Al-Sagri, 2015) إلى أن تكوين كولنيري يكون مناظراً لأحداث فقدان الأوكسجين العالمية (OAE-2) من خلال الثذوذ الموجب لنظير الكاربون العضوي ومحتوى الكاربون العضوي الكلي العالي وخصائص ومكونات المادة العضوية من خلال إستخدام تقنية تقييم الصخور للإنحلال الحراري (Rock-Eval pyrolysis)، وقد أشار إلى ضرورة تحديد أو حصر عمر التكوين بالسينوماني المتأخر-التوروني المبكر Latest .(Cenomanian-Early Turonian تم تحديد وإعتماد عمر تكوين كولنيري في الدراسة الحالية بالتوروني المبكر (Early Turonian) باستخدام متحجرات النانو الكلسية (Al-Lhaebi et al., 2020). ويعد تكوين كولنيري واحدا من التكوينات التي ترسبت ضمن الأحداث التكتونية الرئيسة للصفيحة العربية التي تُعد فترة مهمة وذلك بسبب (Al-Sharhan and Nairn, 1988) (Tectonic Megasequence" AP.9") وجود الهيدروكاربونات في الخزانات النفطية الكاربوناتية في عُمان والإمارات العربية المتحدة وقطر والكويت وإيران والعراق (Hollis and Sharp, 2011). ومن المظاهر المهمة خلال فترة الطباشيري الأوسط هي موقع القارات القديمة، إذ تجمعت الكتل القارية في قارتين عظميتين هما قارة لوراسايا (Laurasia) وقارة كوندوانا (Gondwana) وكان محيط التيش آنذالك يقع في خطوط العرض الدنيا ويتوسع نحو الجهة الشرقية مما أدى إلى حصر الإتصال الثمالي-الجنوبي بين الأحواض المحيطية (Giorgioni, 2012)، وكذلك أدى إلى جعل التيارات المحيطية العالمية أن تكون بإتجاهات متوازية Barron and Peterson, 1989; Föllmi and Delamette, 1991; Poulsen et ) وقريبة من خط الاستواء .(al., 1998

اشتملت الدراسة الحالية على ثلاثة مقاطع سطحية لتكوين كولنيري في شمال شرقي العراق، يقع مقطعان منها في محافظة السليمانية وهما مقطعا أزمر ودوكان ويقع المقطع الثالث (مقطع ديجلة) في محافظة أربيل. وتقع Low and High Folded ) جميع المقاطع المدروسة من الناحية التكتونية ضمن نطاقي الطيات الواطئة والعالية 
(Zones) في الاتجاه الغربي لحزام زاكروس (Zagros Fold-Thrust belt) (الثكل 2). وفيما يلي وصف مواقع تلك المقاطع:

مقطع ديجله Degala section: يقع هذا المقطع على بعد 36 كيلومترا شمال شرقي مدينة أربيل بين قرية ديجله وقرية علياوا في الجناح الجنوبي لطية بينا باوي (Bina-Bawi Anticline) المحدبة عند خط طول $44^{\circ}$

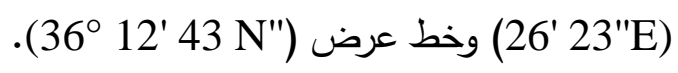

مقطع دوكان Dokan section: يقع هذا المقطع في طية كوسرت المحدبة (Kosrat Anticline) عند

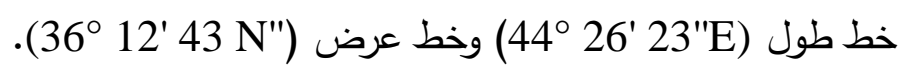

مقطع أزمر Azmir section: يقع هذا المقطع عند تقاطع الطريق الذي يتجه إلى سفح تلة سلتة ري (Salta Re hill)

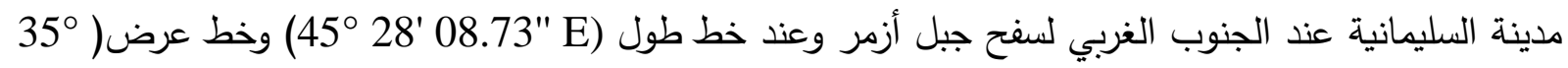
.(36' $13.84 " \mathrm{~N}$
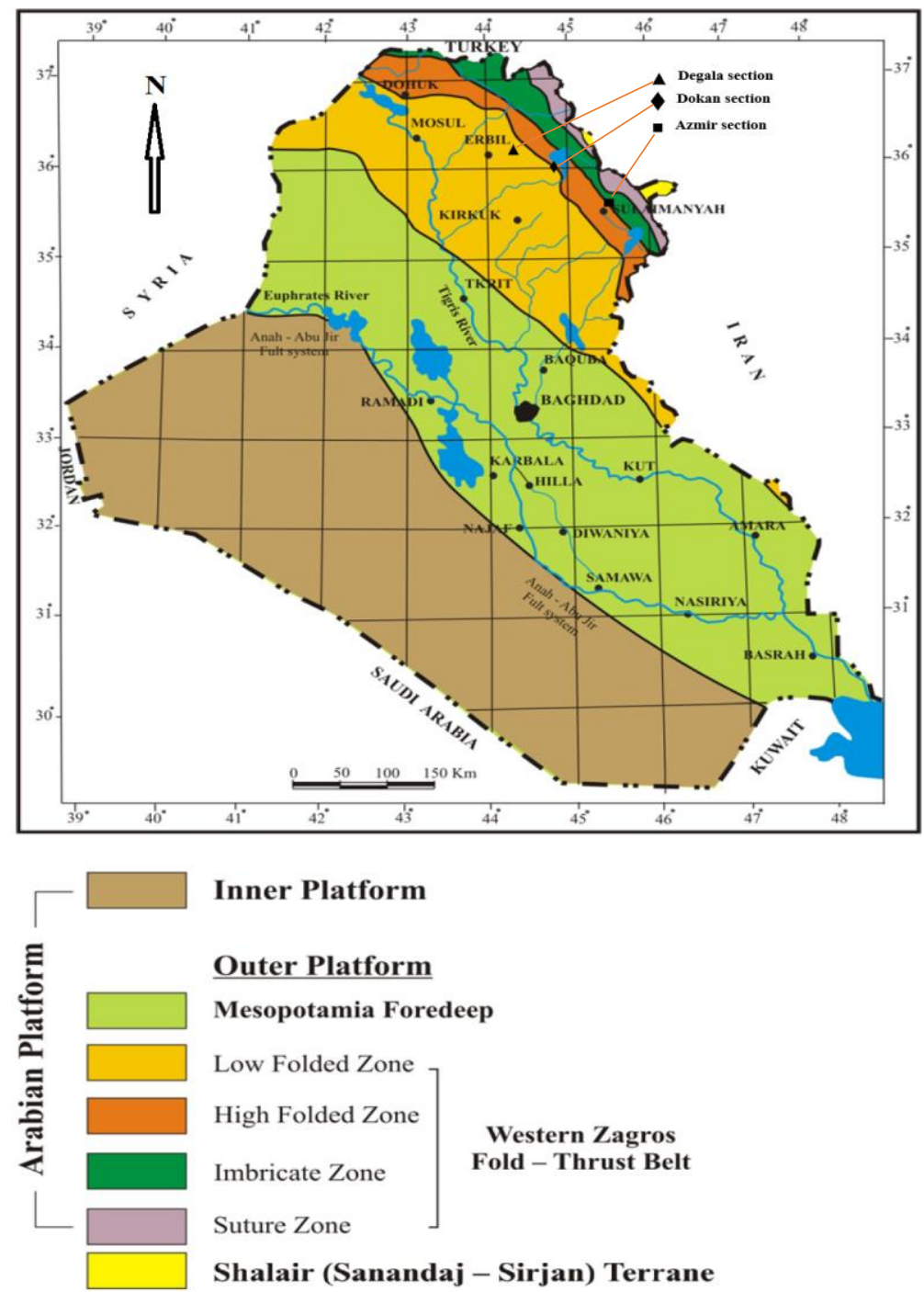

الثكل 2: خارطة العراق التكتونية تبين مواقع مقاطع الدراسة عن (Jassim and Goff, 2006) محورة من قبل .(Fouad, 2015) 


\section{طر ائق العمل}

تم جمع (100) نموذجا صخريا من المنكثفات الصخرية لمقاطع أزمر وديجلة ودوكان لتكوين كولنيري وتم

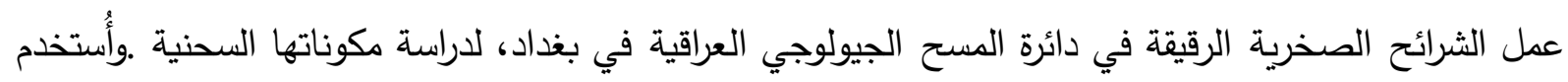
تصنيف (Dunham, 1962) في وصف وتحديد السحنات الدقيقة.

\section{النتائج \\ السحنات الصخرية}

تم تقسيم صخور التكوين إلى عدد من السحنات الصخرية (Lithofacies) إعتماداً على الصفات الكيميائية

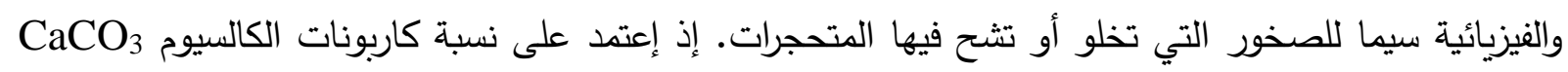

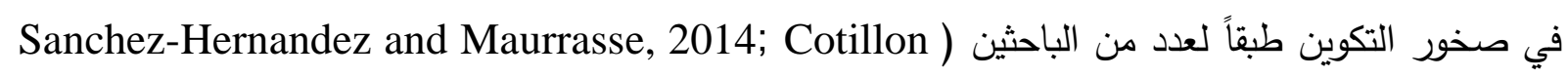

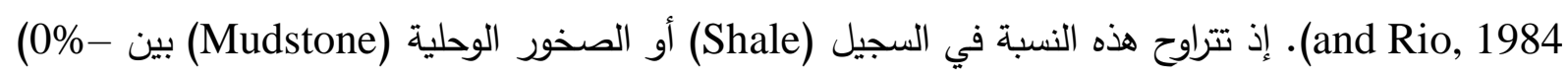

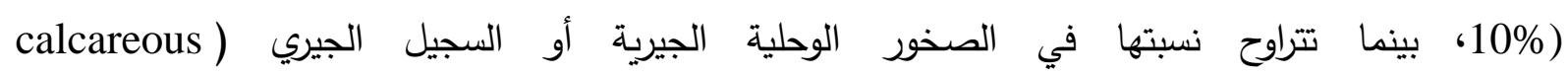
(mudrock/calcareous shale

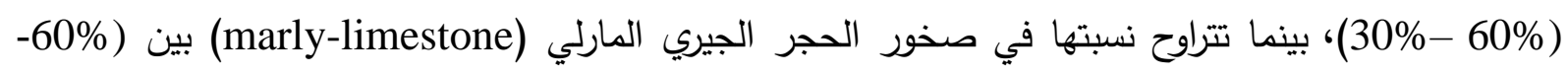

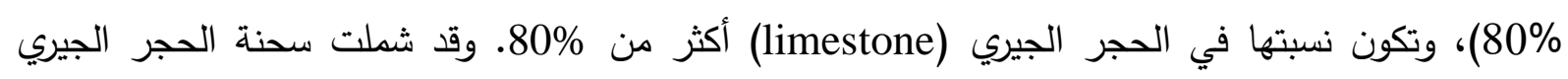
(Limestone lithofacies) التي تضم سحنتي المارل الاحمر المترققة (Laminated red marl lithofacies) وسحنة (Marl lithofacies)

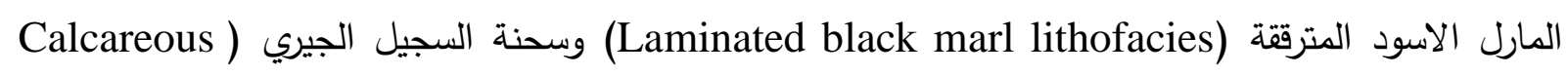
.(shale lithofacies

\section{الوصف الحقلي لمقاطع الدراسة}

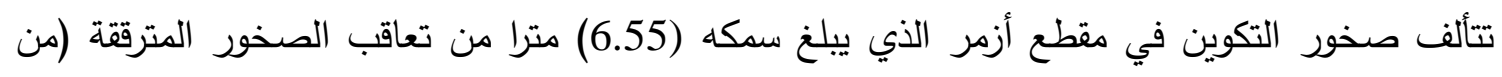

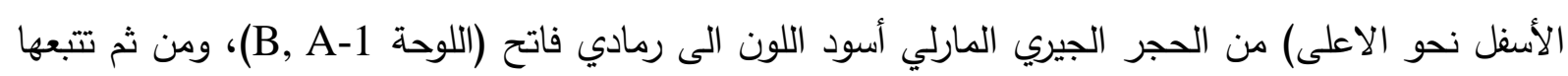

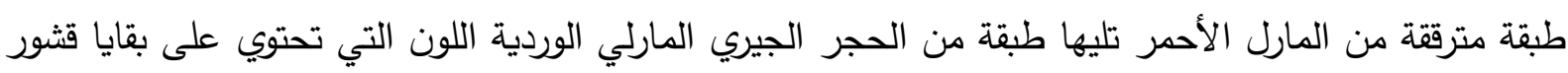

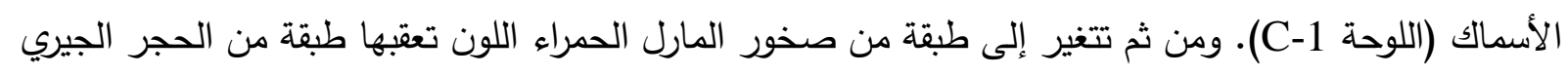

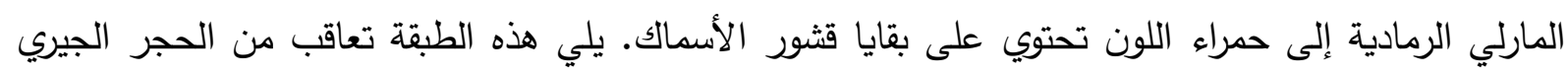

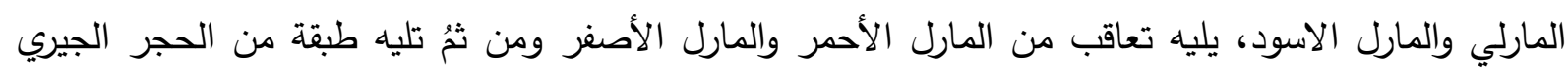

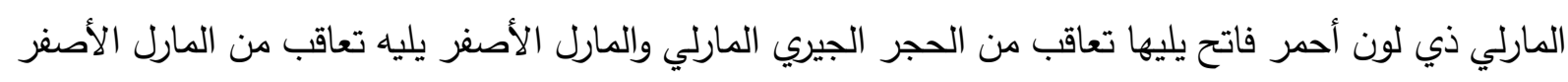

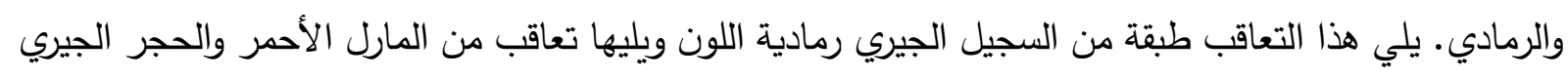

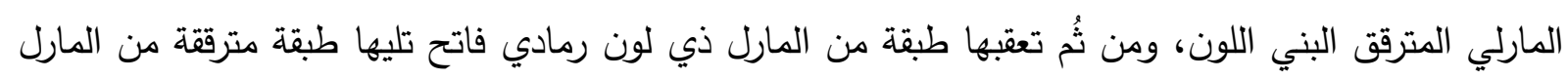

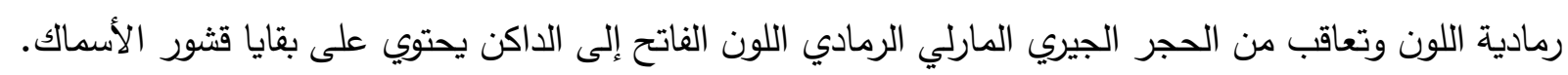

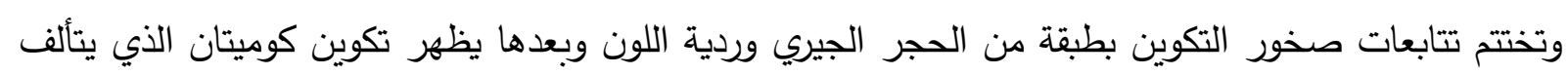
من المارل ذي اللون الرمادي الفاتح. 
بينما تميزت صخور التكوين في مقطع ديجلة الذي يبلغ سمكه 4.2 مترا بلونها الأسود الغني بالمادة العضوية

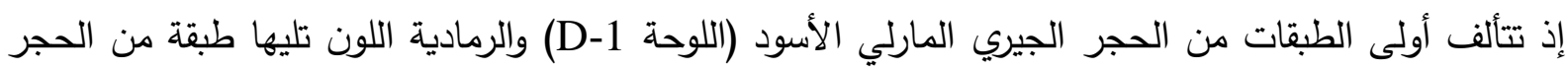
الجيري المارلي المتصفحة الرمادية اللون (اللوحة E-1) تعقبها طبقة من المارل بلون رمادي تليها طبقات مترققة من الحجر الجيري المارلي رمادية وسوداء اللون تعقبها طبقة من الحجر الجيري الاسود يليها تعاقب من الحجر

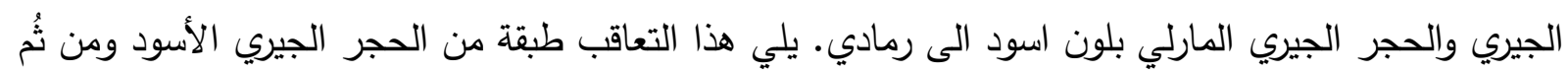

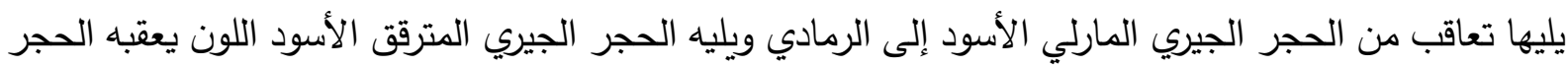

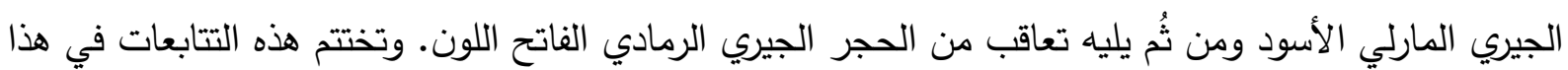
المقطع بتعاقب من الحجر الجيري المارلي الأسود اللون مع الحجر الجيري ذي اللون الرمادي الفاتح. أما مقطع دوكان الذي يكون يسمك (1.46) مترا فيحد تكوين كولنيري من الأسفل تكوين قموقة ومن الأعلى تكوين كوميتان فتمتاز صخوره بصلابتها. وتتألف أولى الطبقات لصخور التكوين في هذا المقطع من طبقتين من المارل الاسود اللون المتصفحة تليها طبقتان من المارل المتصفح على التوالي ومن ثُ تليها طبقة من الحجر الجيري الأسود تعقبها طبقة من الحجر الجيري المارلي، ومن ثُم تليها طبقات متعاقبة من الحجر الجيري الأسود والحجر الجيري المارلي والمارل الأسود. إذ تميزت هذه الطبقات بإحتوائها على حفر الأحياء (Burrows) التي تكون أُفقية إلى مائلة ويبلغ قطرها تقريبا 1cm. وتُختتم التعاقبات لصخور التكوين في هذا المقطع بطبقة من الحجر الجيري الصياء المارلي تحتوي على الكلوكونايت (اللوحة F-1)، لاحظ ايضاً الثكل (3).

\section{السحنات الاقيقة}

تم تمييز ثلاث سحنات أساسية وهي سحنة الحجر الجيري الطيني وسحنة الحجر الجيري الواكي وسحنة الحجر الجيري المرصوص وهي قسمت بدورها إلى عدة سحنات ثانوية. وقد تميزت السحنات الدقيقة باحتوائها على لى لئي الحبيبات الهيكلية التي تضم المنخربات الطافية القزمية من جنس الهيتروهلكس (Heterohelix) الثائعة (اللوحة (C-2 في سحنة الحجر الجيري المرصوص.

وقد لوحظ وجود فتاتات الاصداف النحيفة (Filaments) بهيئة منفردة إذ بلغ معدل طولها 5 مليمترات ومعدل سكها 0.5 مليمترا، وتظهر في سحنات الحجر الجيري الواكي والحجر الجيري المرصوص في مقطع ديجلة (اللوحة E-2). وتم تمييز المنخربات الطافية ذات الحجرات الطولية والتي قد تكون عائدة الى جنس الهيدبركلا في سحنة الحجر الجيري الطيني في مقطع أزمر (اللوحة A-2) وفي سحنة الحجر الجيري الواكي في مقطع ديجلة 

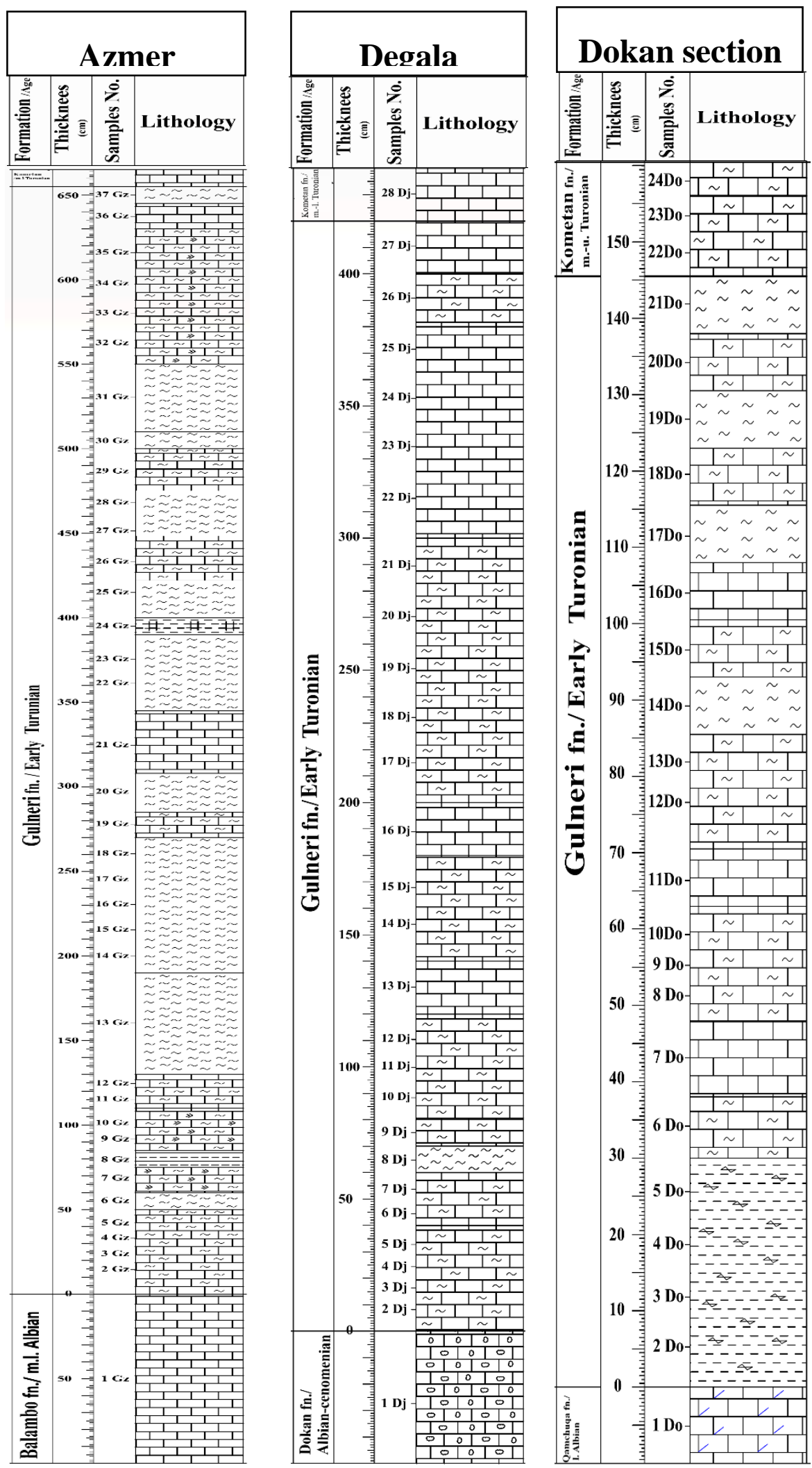

\section{Legend}

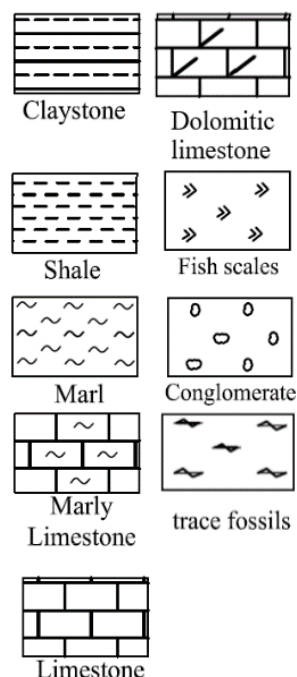

الثكل 3: الأعمدة الرسوبية لمقاطع أزمر وديجلة ودوكان. 
وقد لوحظ وجود وفرة نسبية عالية للراديولاريا في مقطع أزمر مقارنة مع مقطعي دوكان وديجلة. إذ إن

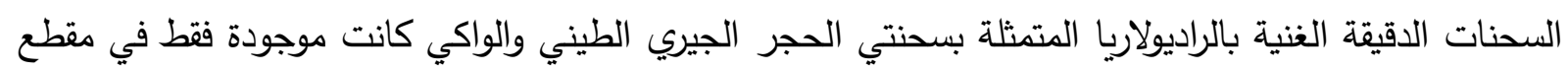
أزمر • وقد تاثرت الراديولاريا بعملية تكون البايرايت سيما في مقطع أزمر، إذ تكون بشكل إحلال لعناصر البناية

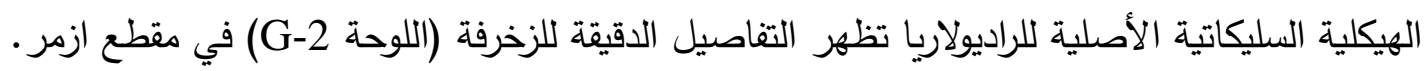
وقد لوحظ وجود الترقق المجهري بين سحنة الحجر الجيري الطيني وسحنة الحجر الجيري الواكي (اللوحة (E-2 فضلا عن تشخيص بقايا عظام السمك الفوسفاتية (اللوحة F-2). كذلك تم تشخيص معدن الكلوكونايت ضمن سحنة الحجر الجيري المرصوص الحاوية على الكلوكونايت الذي تميز بلونه الأخضر الغامق إلى الأخضر الفاتح (اللوحة H-2) في الجزء العلوي من تكوين كولنيري في مقطع دوكان.

\section{المناقثة \\ الأدلة على حلث نقصان الأوكسجين المحيطي-2}

الأدلة الحقلية Field evidences:

يمكن إستتتاج الظروف الأختزالية القديمة للتعاقبات الرسوبية من خلال الملاحظات الحقلية، إذ إفترض

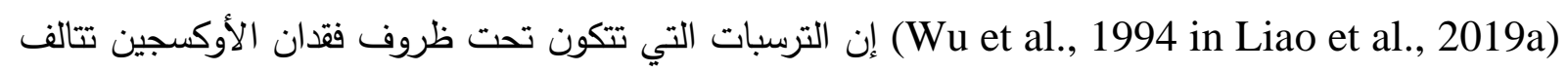
بصورة أساسية من التراكيب المترققة (lamellar structures) أي إنها تحتوي على الترققات (laminations) التي تكون ألوانها داكنة وذات محتوى عالٍ من الكاربون العضوي الكلي وتفتقد إلى الأحياء القاعية والتعكرات الحياتية، بيد انها تحتوي على وفرة من السليكا أو الأحياء الغنية بالفنفور التي تترافق مع ظروف التهات التيارات الصاعدة

.(Liao et al., 2019b; Quijano et al., 2012)

تثير الأدلة الحقلية التي تمثلت بوجود اللون الأسود الداكن إلى الرمادي وكذلك خاصية الترقق والتصفح (اللوحة F E, B, A-1) إلى البيئة المفتقدة للأوكسجين التي تترافق مع فقدان الأحياء القاعية والتعكرات الحياتية.

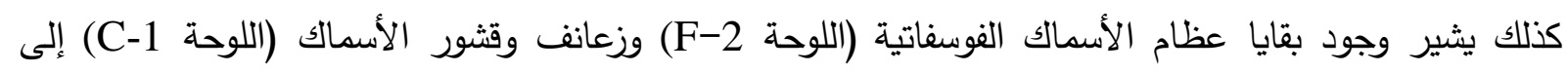
الظروف المفتقدة للأوكسين. ويُشير التورق الموجود في المارل الأحمر المترسب في ظروف بيائية مفتقدة

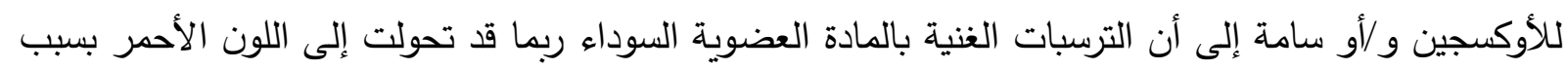
العمليات التحويرية نتيجة أكسدة وتحطم البايرايت (Gertsch et al., 2008).

لا يرتبط وجود الترسبات الحمراء او المحمرة دائما بالمحتوى الأوكسجيني العالي في المياه القاعية، إذ لا يترسب الطين الاحمر في المحيطات الحالية مثل شمالي المحيط الأطلسي رغماً عن وجود الظروف الأوكسيجينة

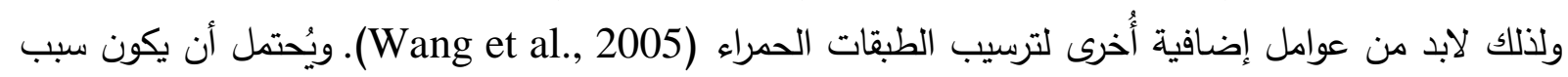
وجود اللون الأحمر في سحنة المارل المترققة في مقطع أزمر هو العمليات التحويرية المبكرة للرواسب الغنية بالمادة العضوية أو أن هذه الرواسب لم تتكون فيها المادة العضوية خلال عمليات الترسيب. 


$$
\text { صفوان فتحي اللهيبي وآخرون }
$$

\section{(1) اللوحة (1)}
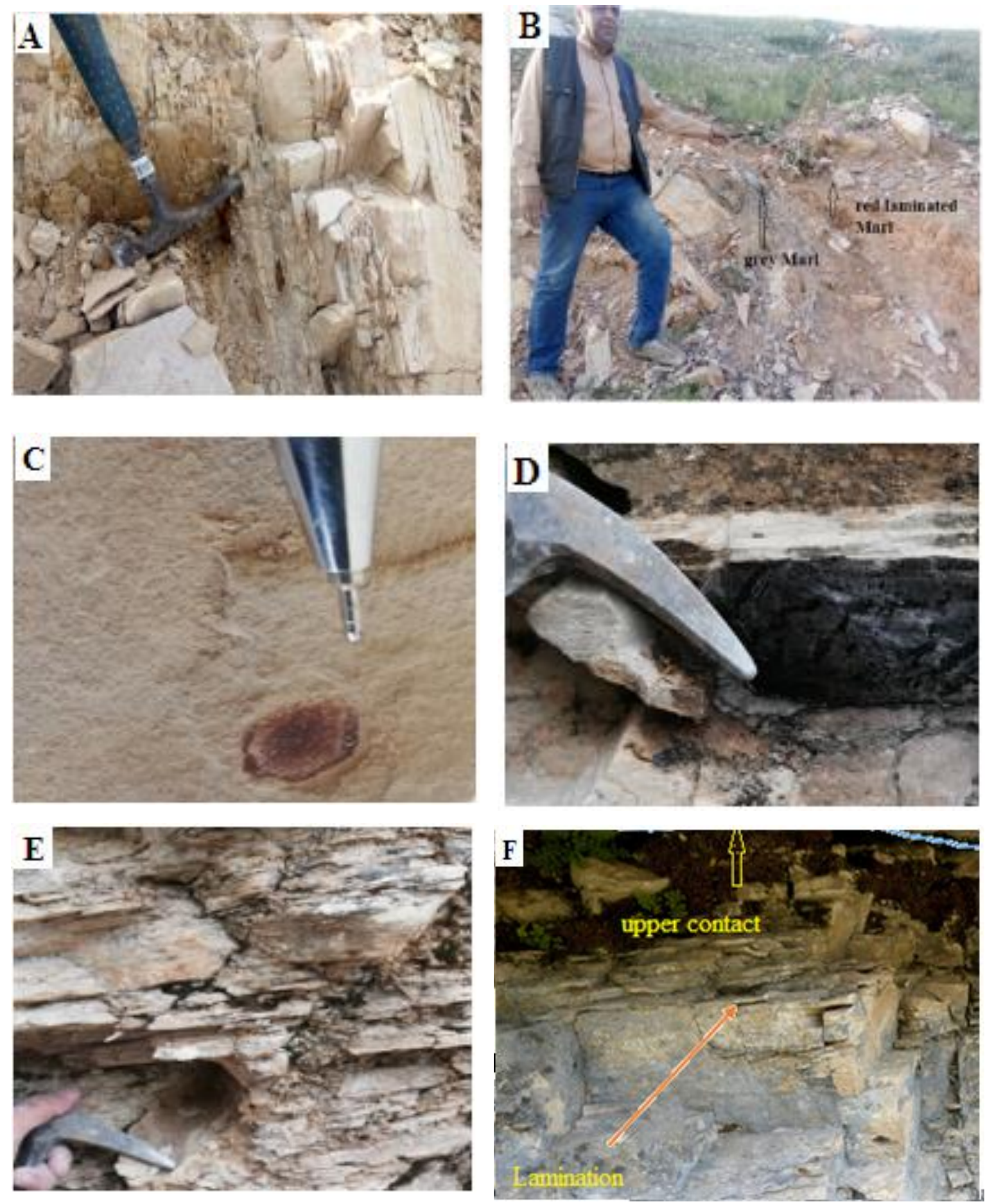

اللوحة 1: صور حقلية لتكوين كولنيري A: التورق والتصفح لصخور المارل الوردية اللون قي مقطع أزمر ، B: تعاقب المارل

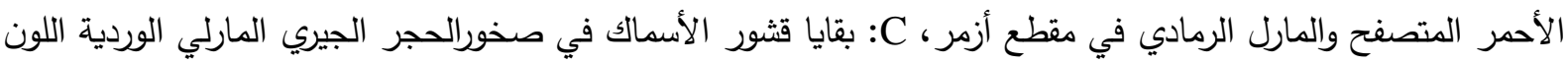

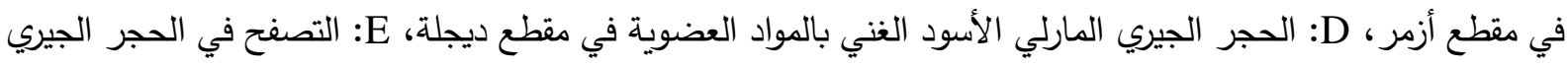

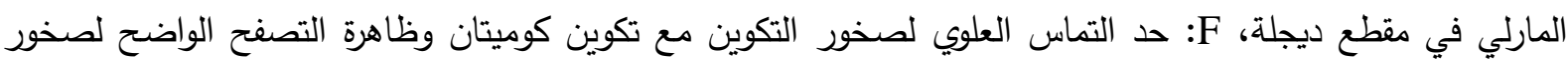

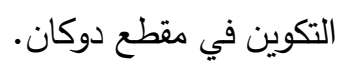




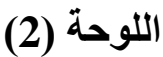
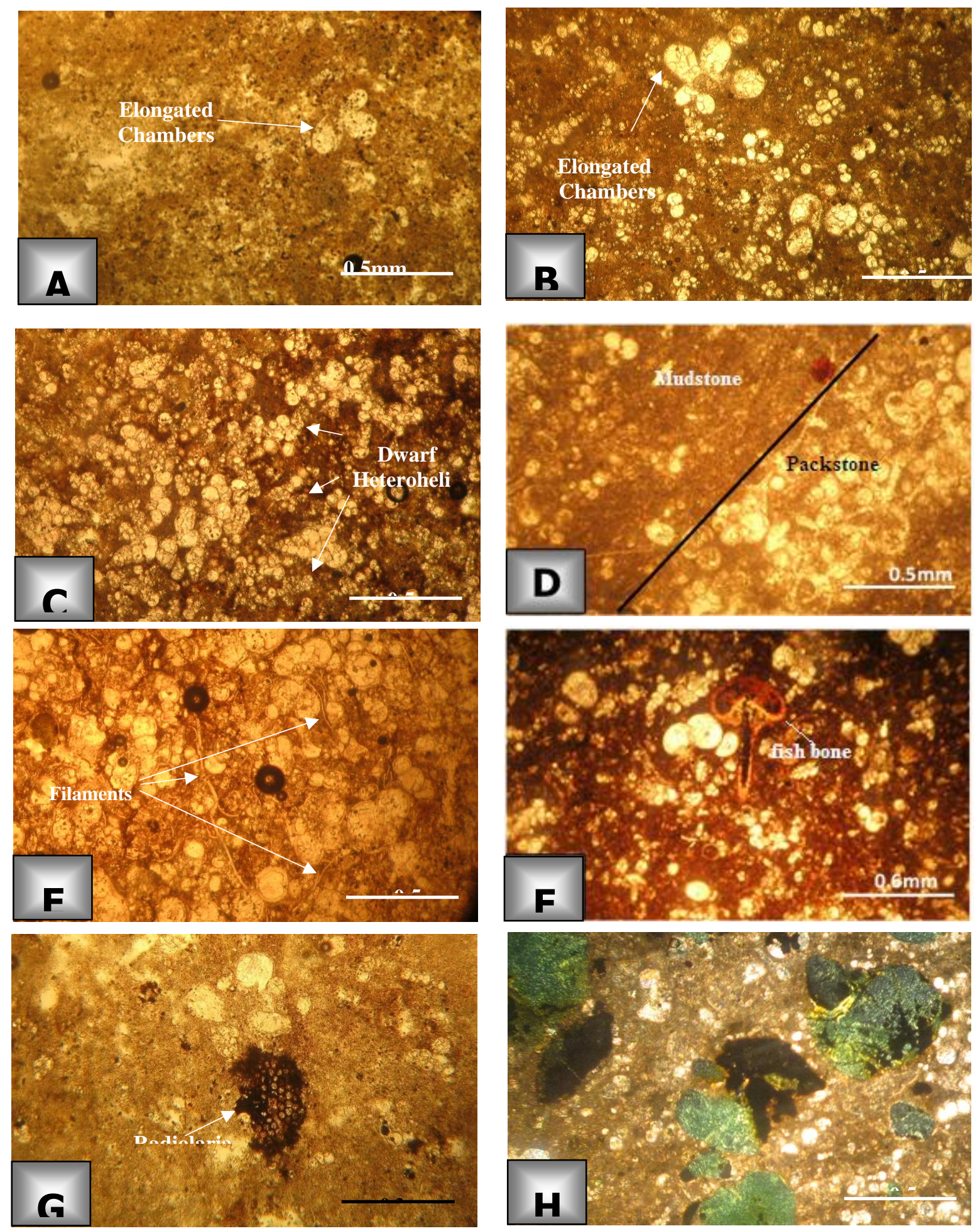

اللوحة (2) الصــور مأخوذة تحت المجهر المسـتقطب، A: المنخربات الطافية ذات الحجرات الطولية في ســنـة الحجر الجيري الطيني، B: المنخربات الطافية ذات الحجرات الطولية في سحنة الحجر الجيري الواكي، C: المنخربات الطافية من جنس الهيتروهلكس القزمية ضمن سحنة الحجر المرصوص، D: الترقق بين سحنتى الحجر الجيري الطيني وسحنة الحجر

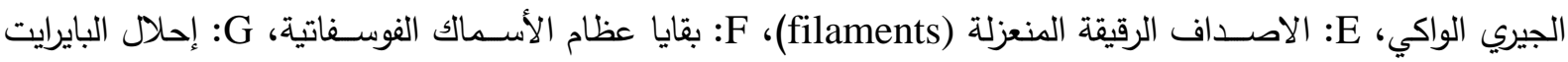

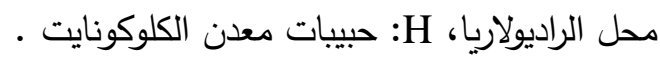


Paleontological evidences الأدلة الحياتية

1 ـ حدث القلمنت Filaments event

تم تشخيص فتاتات الأصداف النحيفة في سحنات الحجر الجيري الواكي والحجر الجيري المرصوص لصخور التكوين في مقطع ديجلة. وتكون هذه الأصداف على هيئة خيوط (stringers) او اهداب (filaments) رفيعة من الكالسايت يكون سُمكها أقل من (5) ملي مايكرون (اللوحة E-2). وتمثل هذه الأصداف في الدراسة الحالية على الأرجح حدث الفلمنت (filaments event). وقد تم توثيق هذه الاصداف عند الحد الزمني الفاصل بين السينوماني والتوروني، ويتم تثخيصها (filaments) عادة عن طريق دراسة الثرائح الرقيقة لنحافة أصدافها ( Denne et)

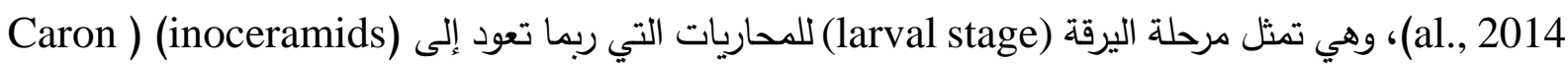
(et al., 2006 ). وقد تم تشخيصها في عدد من المواقع لمحيط التيش عند الحد الفاصل بين السينومانيالتوروني (Negra et al., 2011; Caron et al., 2006). وقد أصبحت أُفقاً طباقياً عالمياً يدعى ( global) filament event التوروني (Caron et al., 2006).

ويتميز هذا الحدث (filaments event) بقة أو ذروة تراكم الأصداف النحيفة (a) filamentous) (allochems بنسبة تتراوح بين (30\%-40\%) (Núñez-Useche et al., 2016). وتدل السحنات الغنية بهذه الأصداف على القرب من الحد الزمني الفاصل بين السينوماني والتوروني (Negra et al, 2011). كما تدل وفرتها قرب هذا الحد على الإنتاجية العضوية العالية نتيجة التغير إلى الظروف المناخية الدافئة الذي أثر بدوره على دوران الكتل المائية. يفسر سبب الوفرة العالية لهذه الاصداف (filaments) عادة كنتيجة للموت الجماعي لليرقات الثنائية الصدفة الطافية ويدل أيضا على البيئة الغنية بالمغذيات (Bomou et al., 2013; Caron et al., 2006). وقد أشار (Negra et al., 2011) إلى أن تركيز هذه الأصداف والذي يُدعى بحدث الفلمنت (filaments event) يُمثل سجلاً للتقدم البحري وتطور الظروف البحرية الطبيعية بعد الفترة الطويلة لفقدان الأوكسجين.

2.

تم تثخيص المنخربات الطافية ذات الحجرات الطولية شعاعية الثكل بنسب قليلة في بعض سحنات الحجر الجيري الطيني والحجر الجيري الواكي في مقطعي ازمر وديجلة (اللوحة B, A-2). وعادة ماتكون هذه المنخربات نادرة في سجل المتحجرات والمحيطات الحالية ولكنها لوحظت بالقرب من الترسبات الغنية بالمادة العضوية لأحداث فقدان الأوكسجين المحيطي (OAEs) (Coccioni et al., 2006, 2007) ). وتثير الإستطالة في الحجرات إلى بالى Kalanat and ) وفرة المغذيات مع قلة الأوكسجين والتكيف إستجابة لظروف إستنزاف الأوكسجين في عمود المياه Vaziri-Moghaddam, 2019 . إذ تعمل الاستطالة على زيادة المساحة السطحية للصدفة التي تُحسن من تبادل الغازات (Coccioni and Luciani, 2004, 2005; Premoli Silvaet et al., 1999).

\section{1. مدث إنحراف الهيتيروهلكس 'Heterohelix shift'}

تميزت المنخربات الطافية الموجودة في سحنتي الحجر الجيري الواكي والحجر الجيري المرصوص لصخور المارل الجيرية والمارل المترققة الغنية بالمادة العضوية في مقطعي ديجلة ودوكان بوفره الهيتروهلسيدا وسيادة جنس لجسي لجري 
الهيتروهلكس (Heterohelix) التي تميزت بأحجامها الناعمة جداً (تقزمها) (اللوحة C-2)، إذ تفتقد فيهما المنخربات ذات الأحجام الطبيعية التي يبلغ حجمها أكبر من 125 ملي مايكرون وكذلك الاحياء القاعية.

تُعد صفة التقزم (dwarfism) للمنخربات الطافية سمة شائعة خلال حدث فقدان الاوكسجين OAE-2 (Coccioni and Luciani, 2004, 2005; Macleod et al., 2000) Coccioni and Luciani, ) (eutrophic conditions) عادة مع ظروف قلة الأوكسجين وأو ظروف التغذية .(2004, 2005

تكون المنخربات القزمية عادة مترافقة مع حدث إنحراف الهيتروهلكس (Heterohelix shift') ولذلك يمكن Gebhardt et ) إستخدام هيمنة جنس كدليل على ظروف الضغط البيئي مثل الدفه المناخي

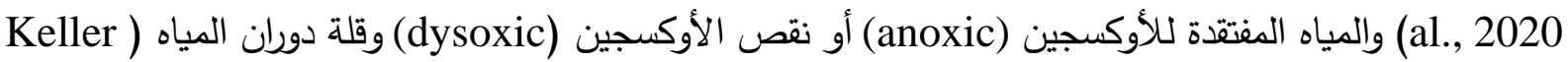
(and Abramovich, 2009; Coccioni and Luciani, 2004; MacLeod et al., 2000 المنخربات القزمية في مقطع ديجلة ودوكان إلى وجود ظروف الضغط البيئي من حيث إرتفاع درجة الحرارة ونقص الأوكسجين وزيادة مستوى غاز ثنائي أوكسيد الكاربون في الجو بينما يكون الوضع (الظروف الطبيعية) مغايراً في السحنات التي تمتلك أحجاما طبيعة للمنخربات.

إن حدث إنحراف الهيتيروهلكس (Heterohelix shift) هو الزيادة السريعة في الوفرة النسبية للهيتروهلسيدا

Nederbragt and (Leckie, 1985) (heterohelicids) Fiorentino, 1999 ). ويعد هذا الحدث حدثاً عالمياً تم توثيقه في أماكن مختلفة من العالم ويعكس إتساع نطاق نقص الأوكسجين (Keller et al., 2008). إن الهيتروهلكس هي من الأجناس التي تتحمل ظروف قلة الأوكسجين، إذ تعكس الزيادة في وفرتها عند الحد بين السينوماني-التوروني في عدة مقاطع مدروسة من العالم تعزيز أو إتساع Keller and Pardo, 2004; Kroon and Nederbragt, 1990; Barrera and ) نطاق نقص الأوكسجين Keller, 1990; Leckie, 1987 (Ifrim, 2006) متكون الزيادة السريعة في وفرة (heterohelicids) مترافقة مع أفضل تكيف لهذه الأحياء مع التغيرات البيئية السريعة من حيث الحرارة والملوحة ومستوى التغذية التي تشير إلى إنخفاض الأوكسجين في عمود المياه (Reolid et al., 2015; Caron et al., 2006).

Coccioni and Luciani, ) تدل الوفرة العالية للهيتروهلسيدا على البيئة الإنتهازية وقلة محتوى الأوكسجين 2004). وتدل المكونات الهيكلية والأرضية لهذه السحنات على الترسيب في ظروف هادئة وفي بيئة الرصيف الإنف العميق والبيئة الحوضية العميقة أو بما يسمى بالرف الخارجي (outer platform) (Flügel, 2010).

\section{Radiolaria الراديولاريا}

توجد السحنات الاقيقة الغنية بالراديولاريا بسحنتي الحجر الجيري الطيني والواكي فقط في مقطع ازمر (اللوحة

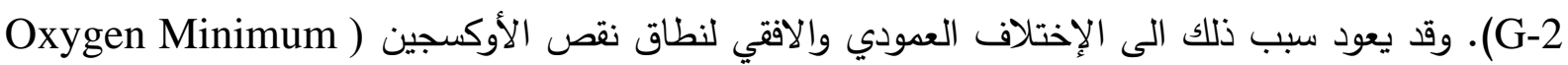
(Zone “OMZ” العضوية في الإتجاه البعيد عن الساحل مُقارنة بالمنطقة القرببة من الساحل التي تمتاز بوفرة الكربونات والمادة 
العضوية (Zhao et al., 2019). يذل وجود الراديولاريا عادة على فترات الإغناء بالمغذيات لمياه المحيطات

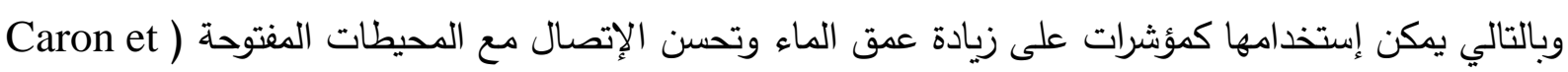

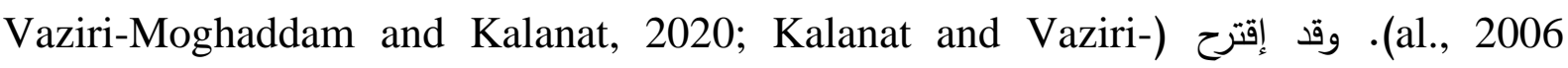
(Moghaddam, 2019 قد ترسبت تحت سطح المياه الغنية بالمغذيات (eutrophication) نتيجة التيارات البحرية الصاعدة وحدوث تهوية ملائمة عند القاع البحري. اذ تتطابق هذه المعطيات مع تكوين كولنيري في مقطع أزمر الذي يفسر وجوده الإتجاه البعيد عن الساحل أي بإتجاه البحر المفتوح. كذلك لوحظ في مقطع أزمر تأثر الراديولاريا بعملية تكوين البايرايت

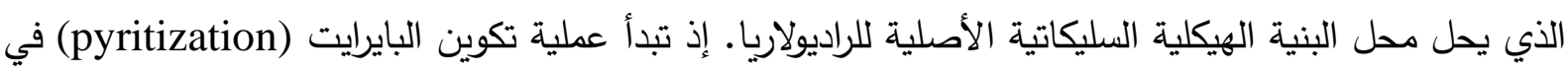
الجزء العلوي من عمود المياه المفتقد للأوكسجين، ويمثل تجلس أو ترسيب (settling) هياكل الراديولاريا (الغنية بالمادة العضوية) مواقع تفكك (decomposition) المادة العضوية وتعزيز إختزال بكتريا الكبريت لإنتاج الكبريتيدات (Sawłowicz and Bąk, 1997).

\section{Fish remains content 3.}

توجد بقايا قشور الأسماك في صخور المارل المتطبقة الحمراء اللون (اللوحة C-1) فضلاً عن وجود البقايا العظمية للأسماك في مقطعي ديجلة ودوكان (اللوحة F-2). تكون بقايا الأسماك في صخور التكوين في مقطع

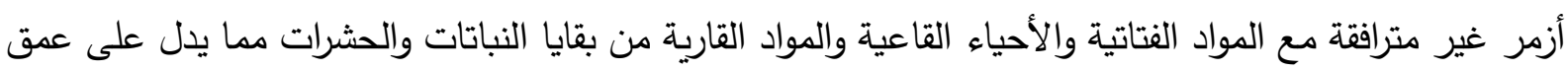
ترسباته في هذا المقطع وبعده عن أية منطقة قارية، بينما يعتقد إن البقايا العظمية للأسماك الصغيرة الحجم في (Karim and Khanaqa, 2017). مقطعي ديجلة ودوكان قد نُقلت خلال العواصف البحرية القوية والأعاصير ويعزى وجود بقايا الأسماك عادة الى الإنتاجية العالية في سطح المياه التي توفر بيئة ملائمة لمعيشة الأسماك وعند موتها تؤدي الظروف المفتقدة للأوكسجين عند القاع الى حفظ بقاياها العظمية. إذ أن الظروف المفتقدة للأوكسجين تكون عادة مناسبة لحفظ بقايا الأسمالك (Leckie et al., 1992)

\section{Mineralogical evidences الأدلة المعدنية}

لوحظ وجود معدن الكلوكونايت في بعض نماذج تكوين كولنيري لاسيما في الجزء العلوي منه في مقطع دوكان والذي يشير إلى التوقف أو البطء في عملية الترسيب. ويُحتمل أن تمثل هذه الطبقة سطح إنغمار (drowning surface) بوجود الكلوكونايت والفوسفات اللذان يدل وجودهما على التكثف (condensation) وقلة معدل الترسيب Amorosi, ) (Amorosi, 1995)

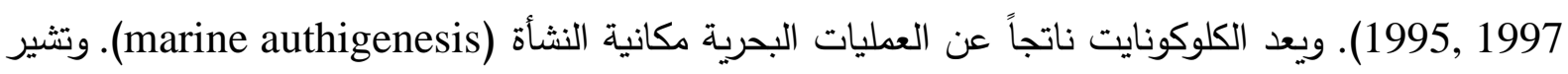
حبيبات الكلوكونايت التي يكون لونها أخضرا غامقا إلى فاتح وتمتلك أحجاماً مُختلفة ويحتوي بعضها على الثقوق على الأغلب إلى عدم تأثرها بعمليات النقل إلى مسافات بعيدة (Lopez- Quiros et al., 2019)، ويُمكن إستخدام

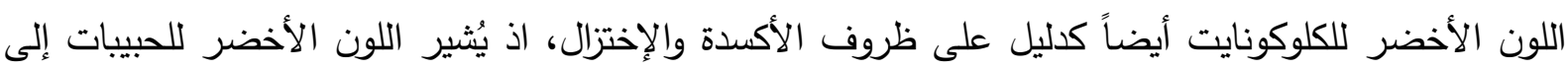
ظروف بيئية أقل أوكسجينية مقارنة باللون الأصفر (Fanning et al., 1989). وفي الدراسة الحالية لم يلاحظ 
ترافق وجود هذا المعدن مع ظواهر الإنكثاف السطحي لإن تكوين كوميتان الذي يعتليه قد ترسب في بيئة لجية حوضية عميقة.

\section{الإستنتاجات}

تبين من خلال الدراسة الحالية إن ترسبات التكوين كانت سوداء إلى رمادية اللون متطبقة ومترققة وتفتقد إلى الأحياء القاعية والتعكرات الحياتية رغماً عن إمتلاك بعض الصخور في مقطع أزمر ألوانا فاتحة إلى مُحمرة، ولكن وجود العديد من الظواهر الرسوبية والأحيائية والمعدنية تدل على ترسيب التكوين بثكل رئيس في ظروف بيئية مفتقدة للأوكسجين (Anoxic). إذ تمثلت هذه الظواهر بوجود الترققات والبقايا الفوسفاتية للأسماك والمنخربات الطولية الحجرات وإحلال البايرايت محل الراديولاريا فضلاً عن وجود المنخربات القزمية من جنس الهيتروهلكس والأصداف النحيفة للمحاريات ومعدن الكلوكونايت لاسيما ضمن سحنات الحجر الجيري المرصوص. إذ تُشير السحنات المجرية الغنية بالهتروهيلكس إلى حدث إنحراف الهتيروهلكيس (Heterohelix shift)، بينما تُشير السحنات المجهرية الغنية بالأصداف النحيفة إلى حدث الفلمنت (Filaments event). وتُشير هذه الظواهر

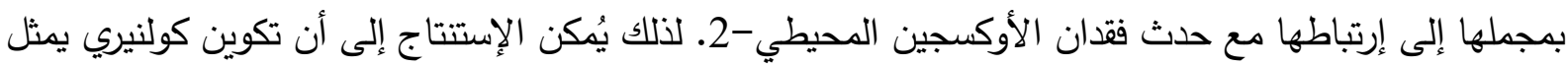
حدث فقدان الأوكسجين المحيطي العالمي-2 عند الحد الفاصل بين السينوماني-التوروني. المصادر

Al-Lhaebi, S.F., Al-Badrani, O.A., Al-Juboury, A.I. and Mahanipour, A., 2020. Paleoclimatic insights on the Cenomanian-Turonian oceanic anoxic event (OAE2) from northern Iraq based on calcareous nannofossils and geochemical data. Iraqi Geological Journal, Vol. 53, pp. 68-86.

Al-Sagri, K.E.A., 2015. Linking the Timing of Deposition and Organic Matter Richness of the Gulneri Formation of Northern Iraq to the Global Oceanic Anoxic Event 2 (OAE 2): Implications to better constrain the Depositional Models of Iraqi's Oil Source Beds and their Timing of Deposition. Iraqi Journal of Science, Vol. 56, No. 3A, pp. 2007-2023.

Al-Sharhan, A.S. and Nairn, A.E.M., 1988. A review of the cretaceous formations in the Arabian Peninsula and gulf: part II. Mid-Cretaceous (Wasia group) stratigraphy and palaeogeography. Journal of Petroleum Geology, Vol. 11, pp. 89112.

Amorosi, A., 1995. Glaucony and sequence stratigraphy: a conceptual framework of distribution in siliciclastic sequences. J. Sediment. Res., Sect. B Stratigr. Glob. Stud., Vol. 65, pp. 419-425.

Amorosi, A., 1997. Detecting compositional, spatial and temporal attributes of glaucony, a tool for provenance research. Sediment. Geol., Vol. 109, pp. 135-153.

Arthur, M.A. and Premoli-Silva, I., 1982. Development of widespread organic carbonrich strata in Mediterranean Tethys. In: Nature and Origin of Cretaceous Carbonrich Facies (Schlanger, S.O. and Cita, M.B., Eds.). Academic Press, London, pp. 7-54. 
Arthur, M.A., Dean, W. E. and Pratt, L. M., 1988. Geochemical and climatic effects of increased marine organic burial at the Cenomanian-Turonian boundary. Nature, Vol. 335, pp. 714-717.

Barrera, E., Keller, G., 1990. Foraminiferal stable isotope evidence for gradual decrease of marine productivity and Cretaceous species survivorship in the earliest Danian. Paleoceanography and Pleoclimatology, Vol. 5, pp. 867-890.

Barron E.J., Peterson, W. H., 1989. Model Simulation of the Cretaceous Ocean Circulation. Science, Vol. 244, pp. 684-686.

Bellen, R.C. Van. Dunnington, H.V., Wetzel, R., Morton, D., 1959. Lexique Stratigraphique International, V.III: Asie, Fasc. 10 a, Iraq, 333p.

Berner, R.A., 2001. Modeling atmospheric O2 over Phanerozoic time. Geochimica et Cosmochimica Acta, Vol. 65, pp. 685-694.

Bomou, B., Adatte, T., Tantawy, A.A., Mort, H., Fleitmann, D., Huang, Y. and Föllmi, K.B., 2013. The expression of the Cenomanian-Turonian oceanic anoxic event in Tibet. Palaeogeography, Palaeoclimatology, Palaeoecology, Vol. 369, pp. 466481.

Buday, T., 1980. The Regional Geology of Iraq, Vol. 1, Stratigraphy and Palaeogeography, Dar Al-Kutub Publishing House. Mosul University. Mosul, Iraq, 445p.

Caron, M., Dall'Agnolo, S., Accarie, H., Barrera, E., Kauffman, E. G., Amédro, F. and Robaszynski, F. 2006. High-resolution stratigraphy of the Cenomanian-Turonian boundary interval at Pueblo (USA) and wadi Bahloul (Tunisia): stable isotope and bio-events correlation. Geobios, Vol. 39, pp. 171-200.

Caron, M., Robazynski, F., Amedro, F., Baudin, F., Deconinck, J.F., Hochhuli, P., von Salis-Perch Nielsen, K. and Tribovillard, N., 1999. Estimation de la duree de l'evenement anoxique global au passage Cenomanien/Turonien; approche cyclostratigraphique dans la formation Bahloul en Tunisie centrale. Bulletin de la Société géologique de France, Vol. 170, pp. 145-160.

Coccioni, R. and Luciani, V., 2004. Planktonic foraminifera and environmental changes across the Bonarelli Event (OAE-2, latest Cenomanian) in its type area: a highresolution study from the Tethyan reference Bottaccione section (Gubbio, Central Italy). Journal of Foraminiferal Research, Vol. 34, pp. 109-129.

Coccioni, R. and Luciani, V., 2005. Planktonic foraminifers across the Bonarelli Event (OAE-2, latest Cenomanian): the Italian record. Palaeogeography, Palaeoclimatology, Palaeoecology, Vol. 224, pp. 167-185.

Coccioni, R., Luciani, V. and Marsili, A., 2006. Cretaceous anoxic events and radially elongated chambered planktonic foraminifera: paleoecological and paleoceanographic implications. Palaeogeography, Palaeoclimatology, Palaeoecology, Vol. 235, pp. 66-92.

Coccioni, R., Premoli Silva, I., Marsili, A. and Verga, D., 2007. First radiation of Cretaceous planktonic foraminifera with radially elongate chambers at Angles 
(Southeastern France) and biostratigraphic implications. Rev. Micropaleontology, Vol. 50, pp. 215-224.

Cotillon, P. and Rio, M., 1984. Cyclic sedimentation in the Cretaceous of Deep Sea Drilling Project sites 535 and 540 (Gulf of Mexico), 534 (Central Atlantic) and in the Vocontian Basin (France). Init. Rep. DSDP, Vol. 77, pp. 339-376.

Denne, R.A., Hinote, R.E., Breyer, J. A., Kosanke, T. H., Lees, J. A., EngelhardtMoore, N., Spaw, J. M. and Tur, N., 2014. The Cenomanian-Turonian Eagle Ford Group of South Texas: Insights on timing and paleoceanographic conditions from geochemistry and micropaleontologic analyses. Palaeogeography, Palaeoclimatology, Palaeoecology, Vol. 413, pp. 2-28.

Dunham, R.J., 1962. Classification of carbonate rocks according to depositional textures. In: Ham, W.E. (Ed.), Classification of carbonate rocks a Symposium. American Association of Petroleum Geologists, Memoir, Vol. 1, pp. 108-121.

Fanning, D.S., Rabenhorst, M.C., May, L. and Wagner, D.P., 1989. Oxidation stage of iron in glauconite from oxidized and reduced zones of soil-geologic columns. Clays and Clay Minerals, Vol. 37, pp. 59-64.

Flügel, E., 2010. Microfacies of Carbonate Rocks, Second edition, Springer Heidelberg, $1006 p$.

Föllmi, K.B. and Delamette, M., 1991. Model simulation of Mid-Cretaceous ocean circulation: technical comments. Science, Vol. 251, pp. 94-95.

Fouad, S.F.A., 2015. Tectonic map of Iraq, scale 1: 1000 000, 3rd edition. Iraqi Bulletin of Geology and Mining, Vol. 11, pp. 1-7.

Gebhardt, H., Akande, S.O. and Adekeye, O.A., 2020. Cenomanian to Coniacian sea-level changes in the Lower Benue Trough (Nkalagu Area, Nigeria) and the Eastern Dahomey Basin: palaeontological and sedimentological evidence for eustasy and tectonism. Geological Society, London, Special Publications, Vol. 498, pp. 233-255.

Gertsch, B., Keller, G., Adatte, T., Berner, Z., Kassab, A.S., Tantawy, A.A.A., ElSabbagh, El. and Stueben, D., 2008. Cenomanian-Turonian transition in a shallow water sequence of the Sinai, Egypt. International Journal of Earth Science, Vol. 99, pp. 165-182.

Giorgioni, M., 2012. Long-and short-term changes in Tethyan oceanography and in global carbon cycling during Albian-Cenomanian time. Ph.D. Thesis. ETH Zurich.

Hasegawa, T., 1997. Cenomanian-Turonian carbon isotope events recorded in terrestrial organic matter from northern Japan. Palaeogeography, Palaeoclimatology, Palaeoecology, Vol. 130, pp. 251-273.

Hollis, C. and Sharp, I., 2011. Albian-Cenomanian-Turonian carbonate siliciclastic systems of the Arabian plate: advances in diagenesis, structure and reservoir modeling, (EAGE/Geological Society of London). Petroleum Geoscience, Vol. 17, pp. 207-209.

Ifrim, C., 2006. The Fossil Lagerstätte at Vallecillo, North-Eastern Mexico: Pelagic Plattenkalks related to Cenomanian-Turonian Boundary Anoxia. PhD (ix 151pp): 
Unpublished PhD thesis, University of Karlsruhe, http://digbib.ubka.unikarlsruhe. de/volltexte/1000004528

Ifrim, C., 2015. Fluctuations of the oxygen minimum zone at the end of Oceanic Anoxic Event 2 in the Gulf of Mexico and the response of ammonites. Swiss Journal of Palaeontology, Vol. 134, pp. 217-225.

Jassim, S.Z. and Goff J. C., 2006. Geology of Iraq. Published by Dolin, Prague and Moravian Museum, Berno. 341p.

Jenkyns, H.C., 1980. Cretaceous anoxic events: from continents to oceans. J. Geol. Soc., London, Vol. 137, pp. 171-188.

Kalanat, B. and Vaziri-Moghaddam, H., 2019. The Cenomanian/Turonian boundary interval deep-sea deposits in the Zagros Basin (SW Iran): Bioevents, carbon isotope record and palaeoceanographic model. Palaeogeography, Palaeoclimatology, Palaeoecology, Vol 533, 109238 https://doi.org/10.1016/j.palaeo. $\underline{2019.109238}$

Karim, K.H. and Khanaqa, P.A., 2017. Stratigraphy and Structure of the Southeastern Part of Piramagroon Anticline, Sulaimani Area, Northeast Iraq. Bulletin of the Mineral Research and Exploration, Vol. 154, pp. 27-39.

Karim, K.H., Salih, A.O. and Ahmad, S.H., 2013. Stratigraphic Analysis of AzmirGoizha Anticline by Nannofossils. Journal of Zankoy Sulaimain (JZS), Part A, Vol. 15, pp. 103-124.

Keller, G. and Abramovich, S., 2009. Lilliput effect in late Maastrichtian planktic foraminifera: Response to environmental stress. Palaeogeography, Palaeoclimatology, Palaeoecology, Vol. 284, pp. 47-62.

Keller, G. and Pardo, A., 2004. Paleoecology of the Cenomanian-Turonian Stratotype Section (GSSP) at Pueblo, Colorado. Marine Micropaleontology, Vol. 51, pp. 95-128.

Keller, G., Tantawy, A.A., Berner, Z., Adatte, T., Chellai, E.H. and Stueben, D., 2008. Oceanic events and biotic effects of the Cenomanian-Turonian anoxic event, Tarfaya Basin, Morocco. Cretaceous Research, Vol. 29, pp. 976-994.

Kolonic, S., Wagner, T., Forster, A., Sinninghe Damsté, J.S., Walsworth-Bell, B., Erba, E., Turgeon, S., Brumsack, H.-J., Chellai, E.H., Tsikos, H., Kuhnt, W. and Kuypers, M.M., 2005. Black Shale deposition on the NW African Shelf during the Cenomanian/ Turonian Oceanic Anoxic Event: climate coupling and global organic carbon burial. Paleoceanography, Vol. 20. PA1006, doi: 10.1029/2003PA000950.

Kroon, D. and Nederbragt, A. J., 1990. Ecology and paleoecology of triserial planktic foraminifera. Marine Micropaleontology, Vol. 16, pp. 25-38.

.Kuroda, J., Ogawa, N.O., Tanimizu, M., Coffin, M.F., Tokuyama, H., Kitazato, H. and Ohk-ouchi, N., 2007. Contemporaneous massive subaerial volcanism and Late Cretaceous oceanic anoxic event 2. Earth Planet. Sci. Lett., Vol. 256, pp. 211-223.

Kuypers, M.M., Pancost,R.D. and Sinninghe Damste, J.S., 1999. A large and abrupt fall in atmospheric CO2 concentration during Cretaceous times. Nature, Vol. 399, pp. $342-344$. 
Leckie, R.M., 1985. Foraminifera of the Cenomanian-Turonian boundary interval, Greenhorn Formation, Rock Canyon Anticline, Pueblo, Colorado. In Fine-grained deposits and biofacies of the Cretaceous Western Interior Seaway-evidence of cyclic sedimentary processes (eds. Pratt, L., Kauffman, E. \& Zelt, F.), Society of Economic Paleontologists and Mineralogists, Field Trip Guidebook, Vol. 4, pp.139-150.

Leckie, R.M., 1987. Paleoecology of mid-Cretaceous planktonic foraminifera: a comparison of open ocean and epicontinental sea assemblages. Micropaleontology, Vol. 33, pp. 164-176.

Leckie, D.A., Singh, C., Bloch, J., Wilson, M., Wall, J., 1992. An anoxic event at the Albian -Cenomanian boundary: the Fish Scale Marker Bed, northern Alberta, Canada. Palaeogeography, Palaeoclimatology, Palaeoecology, Vol. 92, pp. 139-166.

Liao, Z.W., Hu, W.X., Cao, J., Wang, X.L. and Hu, Z.Y., 2019a. Petrologic and geochemical evidence for the formation of organic-rich siliceous rocks of the Late Permian Dalong Formation, Lower Yangtze region, southern China. Marine and Petroleum Geology, Vol. 103, pp. 41-54.

Liao, Z. W., Hu, W.X., Fu, X.G. and Hu, Z.Y., 2019b. Geochemistry of upper Permian siliceous rocks from the Lower Yangtze region, southeastern China: implications for the origin of chert and Permian ocean chemistry. Petroleum Science, Vol. 16, pp. 252-266.

Lopez-Quiros, A., Escutia, C., Sanchez-Navas, A., Nieto, F., Garcia-Casco, A., MartinAlgarra, A., Evangelinos, D. and Salabarnada, A., 2019. Glaucony authigenesis, maturity and alteration in the Weddell Sea: an indicator of paleoenvironmental conditions before the onset of Antarctic glaciation. Sci. Rep. Vol. 9, pp. 1-12.

Macleod, N., Ortiz, N., Fefferman, N., Clyde, W., Schulter, C. and Maclean, J., 2000. Phenotypic response of Foraminifera to episodes of global environment change. In: Culver, S.J., Raw-son, P. (Eds.), Biotic Response to Global Change: The Last 145 Million Years. Cambridge University Press, Cambridge, pp. 51-78

Nederbragt, A.J. and Fiorentino, A., 1999. Stratigraphy and palaeoceanography of the Cenomanian-Turonian Boundary Event in Oued Mellegue, north-western Tunisia. Cretaceous Research, Vol. 20, pp. 47-62.

Negra, M.H., Zagrarni, M.F., Hanini, A. and Strasser, A., 2011. The filament event near the Cenomanian-Turonian boundary in Tunisia: filament origin and environmental signification. Bulletin. Société Géologique de France, Vol. 182, pp. 507-519.

Nielsen, A.T., Schovsbo, N.H., 2011. The Lower Cambrian of Scandinavia: Depositional environment, sequence stratigraphy and palaeogeography. Earth-Sci. Rev. vol.107, pp.207-310.

Núñez-Useche, F., Canet, C., Barragán, R. and Alfonso, P., 2016. Bioevents and redox conditions around the Cenomanian-Turonian anoxic event in Central Mexico: Palaeogeography, Palaeoclimatology and Palaeoecology, Vol. 449, pp. 205-226. 
Paul, C.R.C., Lamolda, M.A., Mitchell, S.F., Vaziri, M.R., Gorostidi, A. and Marshall, J.D., 1999. The Cenomanian-Turonian boundary at Eastbourne (Sussex, UK): a proposed European reference section. Palaeogeography, Palaeoclimatology, Palaeoecology, Vol. 150, pp. 83-121.

Poulsen C.J., Seidov D., Barron, E.J. and Peterson, W.H., 1998. The impact of paleogeographic evolution on the surface oceanic circulation and the marine environment within the mid-Cretaceous Tethys: Paleoceanography, Vol. 13, pp. 546-559.

Premoli Silva, I., Erba, E., Salvini, G., Locatelli, C. and Verga, D., 1999. Biotic changes in Cretaceous oceanic anoxic events of the Tethys. Journal of Foraminiferal Research, Vol. 29, pp. 352-370.

Pucéat, E., Lecuyer, C., Sheppard, S.M.F., Dromart, G., Reboulet, S. and Grandjean, P., 2003. Thermal evolution of Cretaceous Tethyan marine waters inferred from oxygen isotope composition of fish tooth enamels. Paleoceanography, Vol. 18, pp. 1-12.

Quijano, M.L., Castro, J.M., Pancost, R.D., de Gea, G.A., Najarro, M., Aguado, Rosales, I. and Martín-Chivelet, J., 2012. Organic geochemistry, stable isotopes, and facies analysis of the Early Aptian OAE- New records from Spain (Western Tethys). Palaeogeography, Palaeoclimatology, Palaeoecology, Vol. 365, pp. 276-293.

Reolid, M., Sánchez-Quiñónez, C.A., Alegret, L. and Molina, E., 2015. Palaeoenvironmental turnover across the Cenomanian-Turonian transition in Oued Bahloul, Tunisia: foraminifera and proxies geochemical. Palaeogeography, Palaeoclimatology, Palaeoecology, Vol. 417, pp. 491-510.

Sageman, B.B., Rich, J., Arthur, M.A., Dean, W.E., Savrda, C.E. and Bralower, T. J., 1998. Multiple Milankovitch cycles in the Bridge Creek Limestone (CenomanianTuronian), Western Interior Basin. SEPM, Concepts in Sedimentology and Paleontology, Vol. 6, pp.153-171.

Sanchez-Hernandez, Y. and Maurrasse, F.J.M., 2014. Geochemical characterization and redox signals from the latest Barremian to the earliest Aptian in a restricted marine basin: El Pui section, Organya Basin, south-central Pyrenees. Chem. Geol., Vol. 372, pp. 12-31.

Sawłowicz, Z. and Bąk M., 1997. Pyritization of Radiolaria in anoxic water column, anoxic deposits of the Cenomanian/Turonian Boundary in the Pieniny Klippen Belt, Poland. Mineral. Slovaca, Vol. 29, pp. 273-274

Schlanger, S.O. and Jenkyns, H.C., 1976. Cretaceous anoxic events: causes and consequences. Geol. Mijnb., Vol. 55, pp. 179-184.

Smrzka, D., Zwicker, J., Kolonic, S., Birgel, D., Little, C.T.S., Marzouk, A.M., Chellai, E.H., Wagner, T. and Peckmann, J., 2017. Methane seepage in a Cretaceous greenhouse world recorded by an unusual carbonate deposit from the Tarfaya Basin, Morocco. The Depositional Record, Vol. 3, pp. 4-37

Snow, L.J., Duncan, R.A. and Bralower, T.J., 2005. Trace element abundances in the Rock Canyon Anticline, Pueblo, Colorado, marine sedimentary section and their 
re-lationship to Caribbean plateau construction and ocean anoxic event 2. Paleoceanography20, PA3005. , doi: 10.1029/2004PA001093.

Soua, M., 2010. Productivity and bottom water redox conditions at the CenomanianTuronian Oceanic Anoxic Event in the southern Tethyan margin, Tunisia. Rev. méditerranéenne de l'environnement, Vol. 4, pp. 653-664.

Soua, M., Zaghbib-Turki, D., Ben Jemia, H., Smaoui, J. and Boukadi, A., 2011. Geochemical record of the Cenomanian-Turonian anoxic event in Tunisia: is it correlative and isochronous to the biotic signal? Acta Geol. Sinica-English Ed., Vol. 85, pp. 1310-1335.

Stein, R., 1990. Organic carbon content/sedimentation rate relationship and its paleoenvironmental significance for marine sediments. Geo-Marine Letters, Vol. 10 , pp. 37-44.

Sugarman, P.J., Miller, K.G., Olsson, R.K., Browning, J.V., Wright, J.D., De Romero, L.M., White, T.S., Muller, F.L. and Uptegrove, J., 1999. The Cenomanian/Turonian carbon burial event, Bass River, NJ, USA: Geochemical, paleoecological, and sea-level changes. Journal of Foraminiferal Research, Vol. 29, pp. 438-452.

Takashima, R., Nishi, H., Hayashi, K., Okada, H., Kawahata, H., Yamanaka, T., Fernando, A.G., and Mampuku, M., 2009. Litho-, bio- and chemostratigraphy across the Cenomanian-Turonian boundary (OAE-2) in the Vocontian Basin of southeastern France. Palaeogeography, Palaeoclimatology, Palaeoecology, Vol. 272, pp. 61-74.

Takashima, R., Nishi, H., Huber, B.T. and Leckie, R.M., 2006. Greenhouse world and the Mesozoic ocean. Oceanography, Vol. 19, pp. 82-92.

Turgeon, S.C. and Creaser, R.A., 2008. Cretaceous oceanic anoxic event 2 triggered by a massive magmatic episode. Nature, Vol. 454, pp. 323-326.

Tyson, R.V. and Pearson, T.H., 1991. Modern and ancient continental shelf anoxia: an overview. In: Tyson, R.V., Pearson, T.H. (Eds.), Modern and Ancient Continental Shelf Anoxia. Geol. Soc. Spec. Publ., Vol. 58, pp. 1-26.

Vaziri-Moghaddam H., and Kalanat, B., 2020. Oxygen level, primary productivity, and water turbulence during the OAE-2 interval of Zagros Basin (SW Iran): Benthic foraminiferal variations in the carbonate microfacies. Gondwana Research, Vol. 83, pp.1-15.

Wang, C.S., Hu, X.M., Sarti, M., Scott, R.W. and Li, X.H., 2005. Upper Cretaceous oceanic red beds in southern Tibet: a major change from anoxic to oxic, deep-sea environments. Cretaceous Research, Vol. 26, pp. 21-32.

Zhao, S.-Z., Li, Y., Min, H.-J., Wang, T., Nie, Z., Zhao, Z.-Z., Qi, J.-Z., Wang, J.-C. and Wu, J.-P., 2019. Development of Upwelling during the Sedimentary Period of the Organic-Rich Shales in the Wufeng and Longmaxi Formations of the Upper Yangtze Region and Its Impact on Organic Matter Enrichment. J. Mar. Sci. Eng., Vol. 7, pp. 99. https://doi.org/10.3390/jmse7040099. 